\title{
Aortic Endograft Infection with Mycobacterium chimaera and Granulicatella adiacens, Switzerland, 2014
}

\author{
Andreas Plate, Thomas A. Kohl, \\ Peter M. Keller, Sabine Majer, \\ Rosamaria Fulchini, Carol Strahm, \\ Cristoforo Medugno, Zoran Rancic, \\ Lars Husmann, Hugo Sax, \\ Stefan Niemann, Barbara Hasse
}

We describe an aortic endograft infection caused by Mycobacterium chimaera and Granulicatella adiacens, successfully treated with prolonged antimicrobial drug therapy after complete explantation of the infected endoprosthesis and extra-anatomical reconstruction. Whole-genome sequencing analysis did not indicate a close relationship to bacterial strains known to cause infections after cardiac surgery.

A ortic endograft infection (AGI) is a serious complication of aortic repair, and treatment involves prolonged antimicrobial drug therapy and complete or partial graft explantation with subsequent in situ or extra-anatomic arterial reconstruction. AGI attributable to nontuberculous mycobacteria (NTM) is a rare condition, and sporadic cases have been described (1). Mycobacterium chimaera is a slowgrowing NTM and a member of the $M$. avium complex. Recent publications show the emergence of disseminated $M$. chimaera infections occurring after open heart surgery (2). A field investigation identified contaminated heater-cooler units (HCUs) as the source of infection $(3,4)$. In addition to valve reconstructions, these cases also involved thoracic aortic grafts. We describe an abdominal AGI caused by M. chimaera and Granulicatella adiacens. Our aim was to find the

Author affiliations: University Hospital Zurich, Zurich, Switzerland

(A. Plate, Z. Rancic, L. Husmann, H. Sax, B. Hasse); Research

Center Borstel-Leibniz-Center for Medicine and Biosciences,

Borstel, Germany (T.A. Kohl, S. Niemann); German Center for

Infection Research, Borstel (T.A. Kohl, S. Niemann); National

Center for Mycobacteria, University of Zurich, Zurich (P.M. Keller);

Institute of Medical Microbiology, University of Zurich, Zurich

(P.M. Keller); Cantonal Hospital Münsterlingen, Münsterlingen,

Switzerland (S. Majer); Cantonal Hospital St. Gallen, St. Gallen,

Switzerland (R. Fulchini, C. Strahm); Cantonal Hospital

Frauenfeld, Frauenfeld, Switzerland (C. Medugno)

DOI: https://doi.org/10.3201/eid2409.180247 source of the M. chimaera infection by using whole-genome sequencing (WGS) to compare the patient's isolate to strains implicated in infections known to occur after cardiac surgery.

\section{The Study}

In March 2014, a formerly healthy 60-year-old man underwent an elective endovascular aortic repair because of an infrarenal aortic aneurysm. In May 2015, the patient sought medical care for low back pain radiating into the left leg. Laboratory examinations showed elevated C-reactive protein $(57 \mathrm{mg} / \mathrm{L}$ [reference range $<5 \mathrm{mg} / \mathrm{L}])$, leukocytosis $(14.8$ $\mathrm{g} / \mathrm{L}$ [reference range $<9 \mathrm{~g} / \mathrm{L}$ ]), and acute kidney injury (estimated glomerular filtration rate $44 \mathrm{~mL} / \mathrm{min}$ [reference range $>80 \mathrm{~mL} / \mathrm{min}]$ ). A ${ }^{18}$ Fluorodeoxyglucose positron emission tomography-computed tomography (PET-CT) examination indicated an AGI and showed an abscess formation in the iliopsoas muscle in close contact with the left common iliac artery; the intraoperative situs was highly suspicious for AGI, including erosion of the left common iliac artery and a visible endograft. The patient was transferred to the University Hospital Zurich (Zurich, Switzerland) for repeat surgery. The surgical procedure entailed complete endoprosthesis removal, closure of the aortic stump below the renal arteries with polypropylene sutures, and omentum coverage. All tissues were debrided, and treatment included vacuumassisted open-abdomen treatment. Perfusion of the lower limbs' arteries was maintained with an axillo-bifemoral reconstruction using a polytetrafluoroethylene graft (Figure 1).

Deep wound cultures obtained during surgical revisions revealed M. chimaera (in 3/3 cultures) and Granulicatella adiacens (in 4/18 cultures). Histopathologic test results were compatible with mycobacterial infection (online Technical Appendix Tables 1, 2, https://wwwnc.cdc. gov/EID/article/24/9/18-0247-Techapp1.pdf). Results of blood cultures and mycobacteriologic blood and sputum cultures remained negative. The patient received a combination therapy containing clarithromycin, rifabutin, ethambutol, and amikacin in the early postoperative phase. After 6 weeks, amikacin was replaced by moxifloxacin. For coverage of $G$. adiacens, amoxicillin was added to the regimen. We treated the patient for a total of 12 months after the extra-anatomic reconstruction. Several PET-CT scans showed a complete metabolic response. 

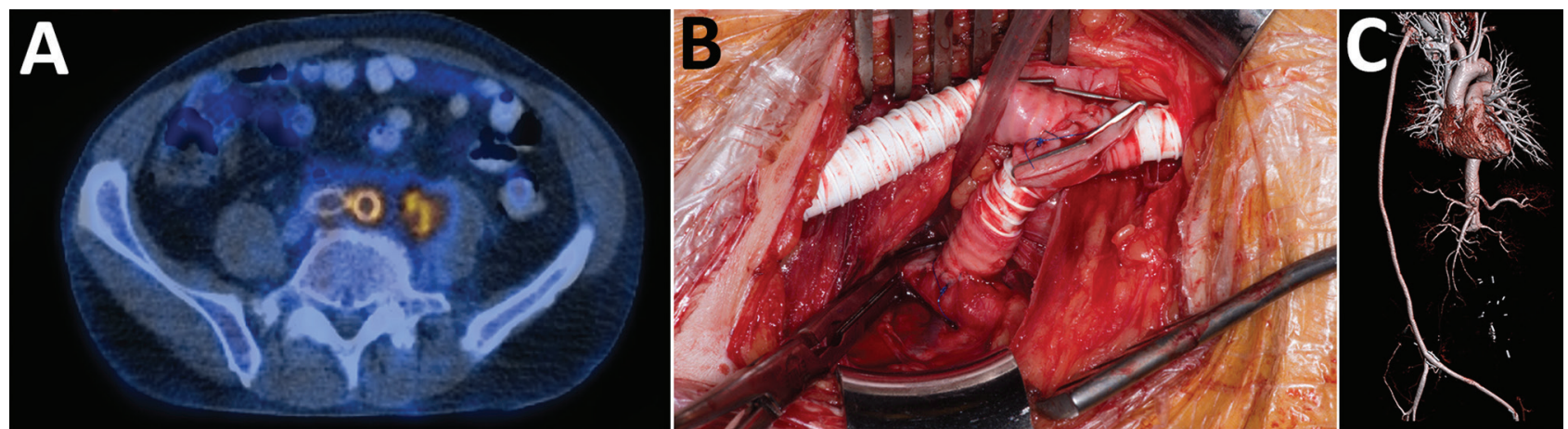

Figure 1. Preoperative, intraoperative, and postoperative images in the case of a patient who received an abdominal aortic endograft and was later diagnosed with Mycobacterium chimaera and Granulicatella adiacens infection, Switzerland, 2014.

A) ${ }^{18}$ Fluorodeoxyglucose positron emission tomography-computed tomography scan at diagnosis indicating a strong, metabolically active (maximum standard uptake value 9.7) aortic endograft infection and an adjacent abscess formation in the iliopsoas in close contact with the left common iliac artery. B) Intraoperative extra-anatomic position of a polytetrafluoroethylene graft through noninfected subcutaneous operative field. C) Satisfactory postoperative result of the axillo-bifemoral bypass on volume-rendered reconstructions of a contrast-enhanced computed tomography.

The diagnostic workup in May 2015 revealed an incidental 5-mm small pulmonary nodulus in the right upper lobe, which was observed to be metabolically active in PET-CT. After recovery from the abdominal intervention, the patient underwent wedge resection, and a localized squamous-cell carcinoma of the lung was confirmed. In April 2016, a relapse of his neoplasia occurred. Despite intensified chemotherapy, the patient died in August 2017 because of progressive pulmonary cancer; no autopsy was performed.

We cultured mycobacteriologic samples in BD MGIT tubes (BD, Franklin Lakes, NJ, USA) on Middlebrook 7H11 agar plates (BD) according to previously published methods (3). Air and water mycobacterial cultures were performed as suggested by the European Centre for Disease Prevention and Control (5).
We analyzed WGS data from the patient's isolate and strains from published studies $(2,6-10)$ by using a reference mapping approach with the M. chimaera DSM-44623 genome (GenBank accession no. NZ_CP015278.1), aided by Burrows-Wheeler Aligner (http://bio-bwa.sourceforge. net), SAMtools (http://samtools.sourceforge.net/cns0. shtml), and GATK (https://software.broadinstitute.org/ gatk) software. We combined variant positions to construct a phylogenetic tree with DnaSP 5.0 (http://www.ub.edu/ dnasp/index_v5.html), FastTree (http://www.microbes online.org/fasttree), FigTree (http://tree.bio.ed.ac.uk/ software/figtree), and EvolView (http://www.evolgenius. info/evolview) software (online Technical Appendix).

The HCU-related outbreak of disseminated M. chimaera infections led us to investigate the hybrid operating

\begin{tabular}{lrrr}
\hline \multicolumn{5}{l}{ Table. Microbiologic test results of air and water samples from the operating room where an abdominal aortic endograft was } \\
performed on a patient later diagnosed with Mycobacterium chimaera and Granulicatella adiacens infection, Switzerland, 2014 \\
\hline \multicolumn{5}{c}{ Sample no. } & Type & Place of sampling & Result \\
\hline 1 & Water & NaCl heater machine & Negative \\
2 & Water & Respirator 1, suction water tank ID 3393 & Negative \\
3 & Water & Respirator 1, breathing hose & Negative \\
4 & Water & Respirator 2, suction water tank & Negative \\
5 & Water & Respirator 2, breathing hose & Negative \\
6 & Water & Operating pre-theater, wash basin, siphon & M. intracellulare* \\
7 & Water & Operating pre-theater, wash basin, cold water & Negative \\
8 & Water & Operating pre-theater, wash basin, hot water & Negative \\
9 & Water & Operating pre-theater, sink, siphon & Negative \\
10 & Water & Operating pre-theater, sink, cistern & Negative \\
11 & Water & Operating pre-theater, sink, cold water & M. paragordonae \\
12 & Water & Negative & Negative \\
13 & Water & Scrub room 2, right side, wash basins 1-3, siphon water & Negative \\
14 & Water & Scrub room 2, right side, wash basins 1-3, after flushing & Negative \\
15 & Water & Scrub room 2, left side, wash basins 4-6, siphon water & Negative \\
16 & Water & Scrub room 2, left side, wash basins 4-6, after flushing & Negative \\
17 & Air & Operating pre-theater, sink, warm water & Negative \\
18 & Air & Air sample 1 & Negative \\
19 & Air & Air sample 2 & Negative \\
20 & Air & Air sample 3 & Negative \\
21 & Air & Air sample 4 & Air sample 5 \\
\hline
\end{tabular}

*Misidentification of M. chimaera excluded by partial $16 \mathrm{~S}$ rDNA sequencing. 


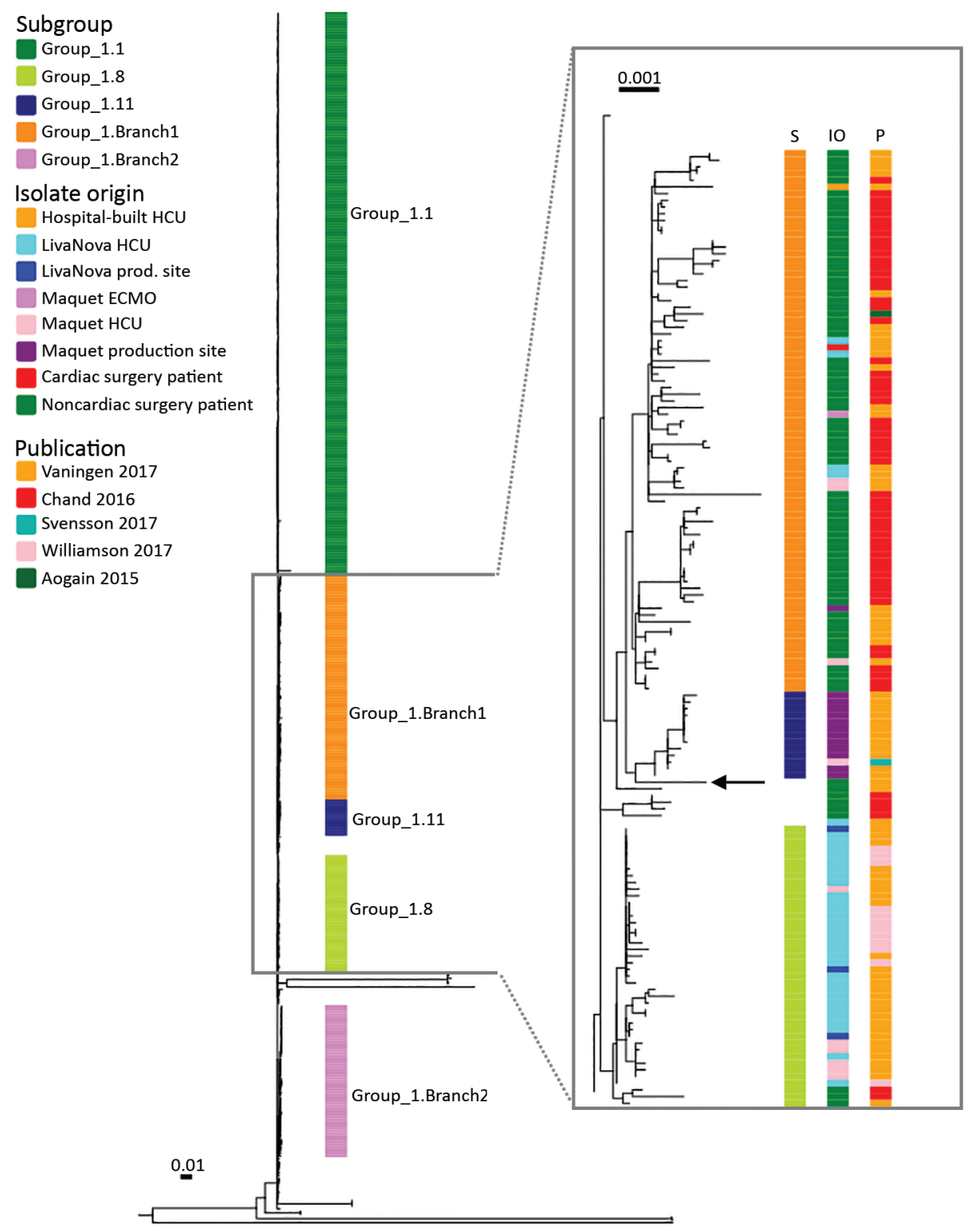

Figure 2. Phylogeny of isolate from case-patient who received an abdominal aortic endograft and was later diagnosed with Mycobacterium chimaera and Granulicatella adiacens infection, Switzerland, 2014, and comparison isolates. Maximum-likelihood tree was built from 14,192 singlenucleotide polymorphism positions of 437 group 1 Mycobacterium chimaera isolates mapped to the DSM-44623 M. chimaera genome (GenBank accession no. NZ_CP015278.1). DSM44623 is shown as a rectangular phylogram with the inferred subgroups indicated. Inset box shows subgroups $1.8,1.11$, and 1.Branch1, annotated with isolate origin and the source publication. Black arrow indicates position of the patient isolate. Group 1.11 consisted mainly of samples collected at the Maquet production site in Rastatt, Germany ( $n=12)$; 1 isolate came from an in-use Maquet HCU. Branch 1 contained primarily strains from patients with pulmonary $M$. chimaera infections $(n=70)$ and strains from LivaNova HCUs $(n=4)$, Maquet HCUs $(n=3)$, Maquet ECMOs $(n=11)$, a hospital-built $\mathrm{HCU}(n=1)$, Maquet production site $(n=1)$, and a patient infected after cardiac surgery $(n=1)$. ECMO, extracorporeal membrane oxygenation; $\mathrm{HCU}$, heater-cooler unit. Scale bars indicate numbers of substitutions per site. room where the patient had undergone his initial surgery (online Technical Appendix Figure). The referring hospital did not use HCUs or extracorporeal membrane oxygenation devices. In summer 2015, we obtained water and air samples from the operating room (Table); results were negative for M. chimaera.

According to a signature single-nucleotide polymorphism-based classification, the patient isolate was similar to the group 1 strains of $M$. chimaera (2). We therefore included all group 1 strains with sufficient WGS data from published studies together with the patient isolate in a combined analysis of a total of 437 strains (Figure 2). The patient isolate did not cluster with subgroup 1.1, which represented all but 1 of the reported cases of disseminated $M$. chimaera infections associated with contaminated HCUs.
Instead, the patient strain clustered with strains from subgroup 1.11 and branch 1 of group 1 (2); however, the patient strain had no close relationship to any other strain included in the comparison.

The endoprosthetic graft (Excluder RMT261214/ PXC121200) of our patient was produced by Gore Medical (Newark, DE, USA). The Swiss Agency for Therapeutic Products submitted a medical device report for the implicated graft to the manufacturer.

\section{Conclusions}

We report an endovascular AGI caused by $M$. chimaera and $G$. adiacens, which was successfully treated with extra-anatomic bypass and prolonged antimicrobial therapy. Because of the histopathology results showing focal 
granulomatous necrotizing inflammation and detection of sparse acid-fast rods in Ziehl Neelsen stain, we outweighed the importance of $M$. chimaera compared with G. adiacens.

Patients at risk for NTM infections are elderly patients with preexisting pulmonary conditions or immunocompromised patients. At AGI diagnosis, the localized pulmonic cancer in this patient was in an early stage, and the patient was not known to be immunocompromised. Blood cultures and repeated sputum specimens were negative for mycobacteria, and PET-CT did not reveal any distant foci. Therefore, we considered a hematogenous spread of a localized and naturally acquired infection to be unlikely. Water and air samples from the operating room were negative for $M$. chimaera; thus, local contamination in the operating room was unlikely. When we compared the patient's isolate with other available $M$. chimaera strains with available WGS data $(2,6-10)$, we observed no association with the cardiac surgery cluster or any other closely related strain in the collection. Because the cardiac surgery cluster originated from $M$. chimaera-contaminated water in medical devices, a contamination of the medical prosthesis at the production site was considered, especially because the poorly soluble polytetrafluoroethylene polymerization is conducted as an emulsion in purified water. However, according to the graft manufacturer, its grafts are produced in a controlled environment, and ethylene oxide gas (EOG) is used for sterilization as recommended by the International Organization for Standardization (standard no. 11135-2007). EOG is widely used because of its good bactericidal activity on many bacterial species and even bacillus spores (11). However, studies showing the effect of EOG on mycobacteria are lacking, and cases of NTM infections caused by inadequate implant sterilization have been reported (12). As the logical next step in the investigation, testing environmental water samples from the production site or from fresh implants for NTM contamination was proposed. However, because of a paperwork assessment, the company decided not to pursue the case further.

Because our investigation involved a single case of an abdominal AGI caused by M. chimaera and G. adiacens, it is too early to draw any conclusions. If further infections emerge, investigations into the adequacy of EOG sterilization for arterial implants should be conducted. In this case, the combination of prolonged antimicrobial therapy, graft explantation, and extra-anatomic reconstruction resulted in sustained healing.

\section{Acknowledgments}

We thank Marisa Kälin, Michael Greiner, and Ivo Fuchs for excellent patient care. We thank Matthias Schlegel for assistance in the outbreak investigation.
The patient was a member of the University Hospital Zurich's Vascular Graft Cohort Study (VASGRA). VASGRA is supported, in part, by grants to B.H. from the Swiss National Science Foundation (grant nos. 32473B_163132/1 and 32473B_163132/2). Parts of the work were funded by the German Center for Infection Research. Members of the VASGRA Cohort Study are (in alphabetical order): Alexia Anagnostopoulos, Barbara Hasse (principal investigator), Lars Husmann, Peter Keller, Bruno Ledergerber, Mario Lachat, Dieter Mayer, Zoran Rancic, Alberto Weber, Rainer Weber, Reinhard Zbinden, and Annelies Zinkernagel.

\section{About the Author}

Dr. Plate is an internal medicine specialist working as an infectious disease fellow in the Division of Infectious Diseases and Hospital Epidemiology, University Hospital Zurich, Switzerland. His primary research interests are epidemiology and foreign body-associated infections.

\section{References}

1. Tsilimparis N, Defreitas D, Debus ES, Reeves JG. Latent Mycobacterium avium infection causing a mycotic suprarenal aortic aneurysm in a human immunodeficiency virus-positive patient. Ann Vasc Surg. 2014;28:1035.e1-4. http://dx.doi.org/ 10.1016/j.avsg.2013.06.036

2. van Ingen J, Kohl TA, Kranzer K, Hasse B, Keller PM, Katarzyna Szafrańska A, et al. Global outbreak of severe Mycobacterium chimaera disease after cardiac surgery: a molecular epidemiological study. Lancet Infect Dis. 2017; 17:1033-41. http://dx.doi.org/10.1016/S1473-3099(17)30324-9

3. Sax H, Bloemberg G, Hasse B, Sommerstein R, Kohler P, Achermann Y, et al. Prolonged outbreak of Mycobacterium chimaera infection after open-chest heart surgery. Clin Infect Dis. 2015;61:67-75. http://dx.doi.org/10.1093/cid/civ198

4. Marra AR, Diekema DJ, Edmond MB. Mycobacterium chimaera infections associated with contaminated heater-cooler devices for cardiac surgery: outbreak management. Clin Infect Dis. 2017;65:669-74. http://dx.doi.org/10.1093/cid/cix368

5. European Centre for Disease Prevention and Control. EU protocol for case detection, laboratory diagnosis and environmental testing of Mycobacterium chimaera infections potentially associated with heater-cooler units: case definition and environmental testing methodology. Stockholm: European Centre for Disease Prevention and Control; 2015 [cited 2018 Jun 27]. https://ecdc.europa.eu/en/ publications-data/eu-protocol-case-detection-laboratory-diagnosisand-environmental-testing

6. Chand M, Lamagni T, Kranzer K, Hedge J, Moore G, Parks S, et al. Insidious risk of severe Mycobacterium chimaera infection in cardiac surgery patients. Clin Infect Dis. 2017;64:335-42. http://dx.doi.org/10.1093/cid/ciw754

7. Perkins KM, Lawsin A, Hasan NA, Strong M, Halpin AL, Rodger RR, et al. Mycobacterium chimaera contamination of heater-cooler devices used in cardiac surgery-United States. MMWR Morb Mortal Wkly Rep. 2016;65:1117-8. http://dx.doi.org/10.15585/mmwr.mm6540a6

8. Svensson E, Jensen ET, Rasmussen EM, Folkvardsen DB, Norman A, Lillebaek T. Mycobacterium chimaera in heater-cooler units in Denmark related to isolates from the United States and United Kingdom. Emerg Infect Dis. 2017;23:507-9. http://dx.doi.org/10.3201/eid2303.161941 
9. Williamson D, Howden B, Stinear T. Mycobacterium chimaera spread from heating and cooling units in heart surgery. N Engl J Med. 2017;376:600-2. http://dx.doi.org/10.1056/NEJMc1612023

10. Mac Aogáin M, Roycroft E, Raftery P, Mok S, Fitzgibbon M, Rogers TR. Draft genome sequences of three Mycobacterium chimaera respiratory isolates. Genome Announc. 2015;3: e01409-15. http://dx.doi.org/10.1128/genomeA.01409-15

11. Mendes GC, Brandão TR, Silva CL. Ethylene oxide sterilization of medical devices: a review. Am J Infect Control. 2007;35:574-81. http://dx.doi.org/10.1016/j.ajic.2006.10.014
12. Robicsek F, Hoffman PC, Masters TN, Daugherty HK, Cook JW, Selle JG, et al. Rapidly growing nontuberculous mycobacteria: a new enemy of the cardiac surgeon. Ann Thorac Surg. 1988; 46:703-10. http://dx.doi.org/10.1016/S0003-4975(10)64742-X

Address for correspondence: Andreas Plate, University Hospital Zurich, Division of Infectious Diseases and Hospital Epidemiology, Raemistrasse 100 CH-8091, Zurich, Switzerland; email: andreas.plate@usz.ch

etymologia

\section{Granulicatella [gran'yoo-lik-ə-tel"ə]}

\section{Ronnie Henry}

I n 1961, Frenkel and Hirsch described strains of streptococci isolated from cases of bacterial endocarditis that grew only in the presence of other bacteria, around which they formed satellite colonies, or in media enriched with sulfhydryl compounds, such as cysteine. These nutritionally variant streptococci were eventually assigned the species Streptococcus defectivus (Latin for "deficient") and $S$. adjacens (because it grows adjacent to other bacteria).

On the basis of later research, these were placed in a new genus Abiotrophia (Greek a, "un-," + bios, "life," + trophe, "nutrition") as A. adiacens and A. defectiva. In 1998 and 1999, 2 additional species of Abiotrophia were described, A. elegans (Latin, "fastidious," referring to fastidious growth requirements) and

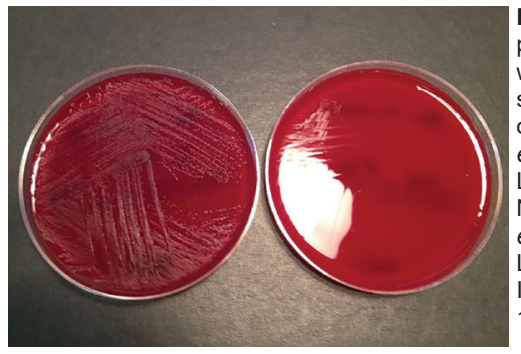

Figure. Blood agar plates with (left) and without (right) pyridoxal supplement from a study of neonatal Granulicatella elegans bacteremia, London, UK. Image from Neonatal Granulicatella elegans Bacteremia, London, UK; Emerging Infectious Diseases Vol. 19 , no. 7 , July 2013.

A. balaenopterae (isolated from a minke whale [Balaenoptera acutorostrata]). In 2000, these new species, along with $A$. adiacens, were reclassified in the new genus Granulicatella (Latin granulum, "small grain," + catella, "small chain").

4. Kawamura Y, Hou XG, Sultana F, Liu S, Yamamoto H, Ezaki T. Transfer of Streptococcus adjacens and Streptococcus defectivus to Abiotrophia gen. nov. as Abiotrophia adiacens comb. nov. and Abiotrophia defectiva comb. nov., respectively. Int J Syst Bacteriol. 1995;45:798-803. http://dx.doi.org/10.1099/ 00207713-45-4-798

5. Lawson PA, Foster G, Falsen E, Sjödén B, Collins MD. Abiotrophia balaenopterae sp. nov., isolated from the minke whale (Balaenoptera acutorostrata). Int J Syst Bacteriol. 1999;49:503-6. http://dx.doi.org/ 10.1099/00207713-49-2-503

6. Roggenkamp A, Abele-Horn M, Trebesius KH, Tretter U, Autenrieth IB, Heesemann J. Abiotrophia elegans sp. nov., a possible pathogen in patients with culture-negative endocarditis. J Clin Microbiol. 1998;36:100-4.

Address for correspondence: Ronnie Henry, Centers for Disease Control and Prevention, 1600 Clifton Rd NE, Mailstop E03, Atlanta, GA 30329-4027, USA; email: boq3@cdc.gov

DOI: https://doi.org/10.3201/eid2409.ET2409 